7 Mueller BU, Pabst T, Osato M, Asou N, Johansen LM, Minden MD et al. Heterozygous PU.1 mutations are associated with acute myeloid leukemia. Blood 2002; 100: 998-1007.

8 Rosenbauer F, Wagner K, Kutok JL, Iwasaki H, Le Beau MM, Okuno Y et al. Acute myeloid leukemia induced by graded reduction of a lineage-specific transcription factor, PU.1. Nat Genet 2004; 36: 624-630.

9 Metcalf D, Dakic A, Mifsud S, Di Rago L, Wu L, Nutt S. Inactivation of PU.1 in adult mice leads to the development of myeloid leukemia. Proc Natl Acad Sci USA 2006; 103: 1486-1491.

10 Stoletov K, Klemke R. Catch of the day: zebrafish as a human cancer model. Oncogene 2008; 27: 4509-4520.

11 Orkin SH, Zon LI. Hematopoiesis: an evolving paradigm for stem cell biology. Cell 2008; 132: 631-644.
12 Dakic A, Metcalf D, Di Rago L, Mifsud S, Wu L, Nutt SL. PU.1 regulates the commitment of adult hematopoietic progenitors and restricts granulopoiesis. J Exp Med 2005; 201: 1487-1502.

13 Pigneux A, Perreau V, Jourdan E, Vey N, Dastugue N, Huguet F et al. Adding lomustine to idarubicin and cytarabine for induction chemotherapy in older patients with acute myeloid leukemia: the BGMT 95 trial results. Haematologica 2007; 92: 1327-1334.

14 Rabbani A, Finn RM, Ausio J. The anthracycline antibiotics: antitumor drugs that alter chromatin structure. Bioessays 2005; 27: 50-56.

15 Anderson KL, Smith KA, Pio F, Torbett BE, Maki RA. Neutrophils deficient in PU.1 do not terminally differentiate or become functionally competent. Blood 1998; 92: 1576-1585.

Supplementary Information accompanies this paper on the Leukemia website (http://www.nature.com/leu)

\title{
OPEN
}

\section{A powerful molecular synergy between mutant Nucleophosmin and Flt3-ITD drives acute myeloid leukemia in mice}

Leukemia (2013) 27, 1917-1920; doi:10.1038/leu.2013.77

Acute myeloid leukemia (AML) is the commonest myeloid malignancy, yet there has been little therapeutic progress for this disease in decades, and only $25-30 \%$ of patients survive long term. ${ }^{1}$ This reflects its pathogenetic complexity and the fact that the molecular basis of its largest cytogenetic subgroup, AML with a normal karyotype (AML-NK), was obscure until recently. Recent advances in DNA sequencing have revealed that AML-NK is molecularly heterogeneous with $>30$ genes recurrently targeted by somatic mutations in this disease. ${ }^{2}$ What is also evident is that each individual case of AML-NK appears to harbor only a small number of coding driver mutations, often as few as three and usually no more than five. ${ }^{2,3}$ Furthermore, it is manifest that the precise combination of driver mutations in the genome of each AML impacts on its salient features, including responsiveness to treatments and prognosis. ${ }^{3}$

These observations provide a sound starting point for systematic mechanistic studies to understand the pathogenesis and improve the treatment of AML-NK. Carefully designed mouse models are the gold standard in the study of normal and malignant hemopoiesis, and are already instructing our understanding of AML-NK. ${ }^{4,5}$ Here, we report that the two most commonly cooccurring somatic mutations in $\mathrm{AML}$, namely Nucleophosmin (NPM1) exon 12 mutations (NPM1C) and internal tandem duplications of FLT3 (FLT3-ITD), cooperate explosively to induce $A M L$ in knock-in mice. In revealing this striking molecular synergy, our work offers a basis for the frequent co-occurrence of these two mutations and provides a valuable model for in-depth studies of the pathogenesis and treatment of this large subgroup of AML.

NPM1 is a nucleolar phosphoprotein involved in many cellular processes. For many of its roles, it relies on its ability to shuttle between the nucleolus, nucleus and cytoplasm using subcellular localization signals. ${ }^{6}$ This ability is impaired in $30 \%$ of AMLs as a result of NPM1C mutations, which disrupt the nucleolar localization signal of NPM1 and generate a nuclear export signal in its place. ${ }^{7}$ Mutant NPM1 is known to bind to and alter the subcellular distribution of several proteins, including HEXIM1, p19Arf and nuclear factor- $\kappa B_{;}^{8}$ however, the relevance of these interactions to $\mathrm{AML}$ is unclear. FLT3-ITD mutations occur in $20-25 \%$ of $\mathrm{AML}^{9}$ and result in ligand-independent receptor dimerization and constitutive FLT3 signaling, ${ }^{10}$ and are associated with an increased risk of relapse. Moreover, patients with low or absent levels of wild-type (WT) FLT3, consistent with loss-of-heterozygosity (LOH) for this locus, have a particularly poor outcome. ${ }^{9}$

Recently, we described a conditional knock-in mouse model of NPM1c mutations and demonstrated that one-third of mice developed delayed-onset $A M L$, suggesting a requirement for cooperating mutations. We went on to show that insertional mutagenesis with transposons led rapidly to $\mathrm{AML}$ in $80 \%$ of $\mathrm{Npm} 1 \mathrm{c}$ mice, in association with specific recurrent mutations including activating insertions in Csf2 and Flt3. ${ }^{4}$ Flt3-ITD homozygous mutant mice exhibit enhanced proliferation and survival properties in hemopoietic progenitors and develop a lateonset disease akin to chronic myelomonocytic leukemia. ${ }^{11}$

To study the combined effects of NPM1C with FLT3-ITD we crossed conditional $\mathrm{Npm} 7^{\text {flox }-\mathrm{CA} /+}$ with constitutive $\mathrm{Flt} 3^{I \mathrm{TD} /+}$ to generate $\mathrm{Npm}^{\text {flox }-\mathrm{CA} /+} ; \mathrm{Flt} 3^{I T D /+}$ double heterozygous mice, then crossed into $\mathrm{Mx} 1-\mathrm{Cre}$ transgenic mice to induce recombination of $N p m 1^{\text {flox }-c A}$ in hematopoietic stem cells. ${ }^{4}$ The Mx1-Cre allele requires induction by interferon, usually achieved by intraperitoneal injection of polyinosinic-polycytidylic acid (plpC). However, we observed universal and rapid emergence of AML (myeloid leukemia with maturation) in uninjected

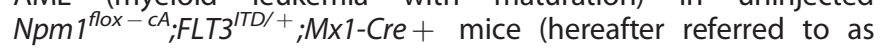
'Npm1C/FIt3-ITD mice'). Mx1-Cre is known to 'leak' in 2-4\% of hemopoietic stem/progenitor cells, ${ }^{12}$ and this was sufficient to rapidly generate $A M L$ from double mutant cells signifying a striking cooperativity between Npm1c and Flt3-ITD. The presence of the cytoplasmic NPM1 was confirmed on protein blots (Figure 1a).

All Npm1 C/Flt3-ITD mice developed AML and became moribund aged 31-68 days (median 49 days; $n=29$ ). By contrast, no case of AML was observed in Npmi flox-CA; $M \times 1-C r e+$ mice (hereafter referred to as 'Npm1c mice'; $n=30$, of which 15 received plpC aged 6-8 weeks), $F L T 3^{I T D /+}$ mice (hereafter referred to as 'Flt3-ITD mice'; $n=34$ ) or WT mice (WT, $n=29$ ) aged to at least 8 months (Figure 1b). Weekly blood counts from 19 mice with each genotype showed a progressive increase in blood leukocyte counts in Npm1c/Flt3-ITD mice, to more than 25-fold that of 
a

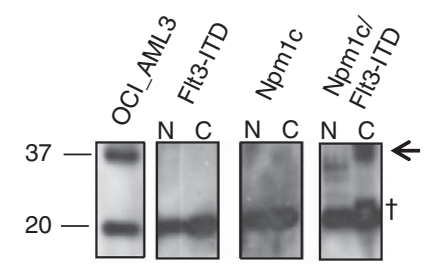

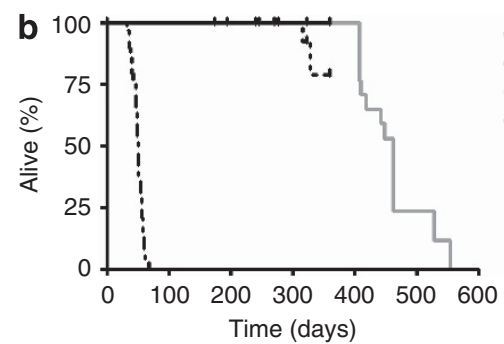

•.• Npm1c/Flt3-ITD

. ... FIt3-ITD

, WT

- Npm1c

$p<0.0001$
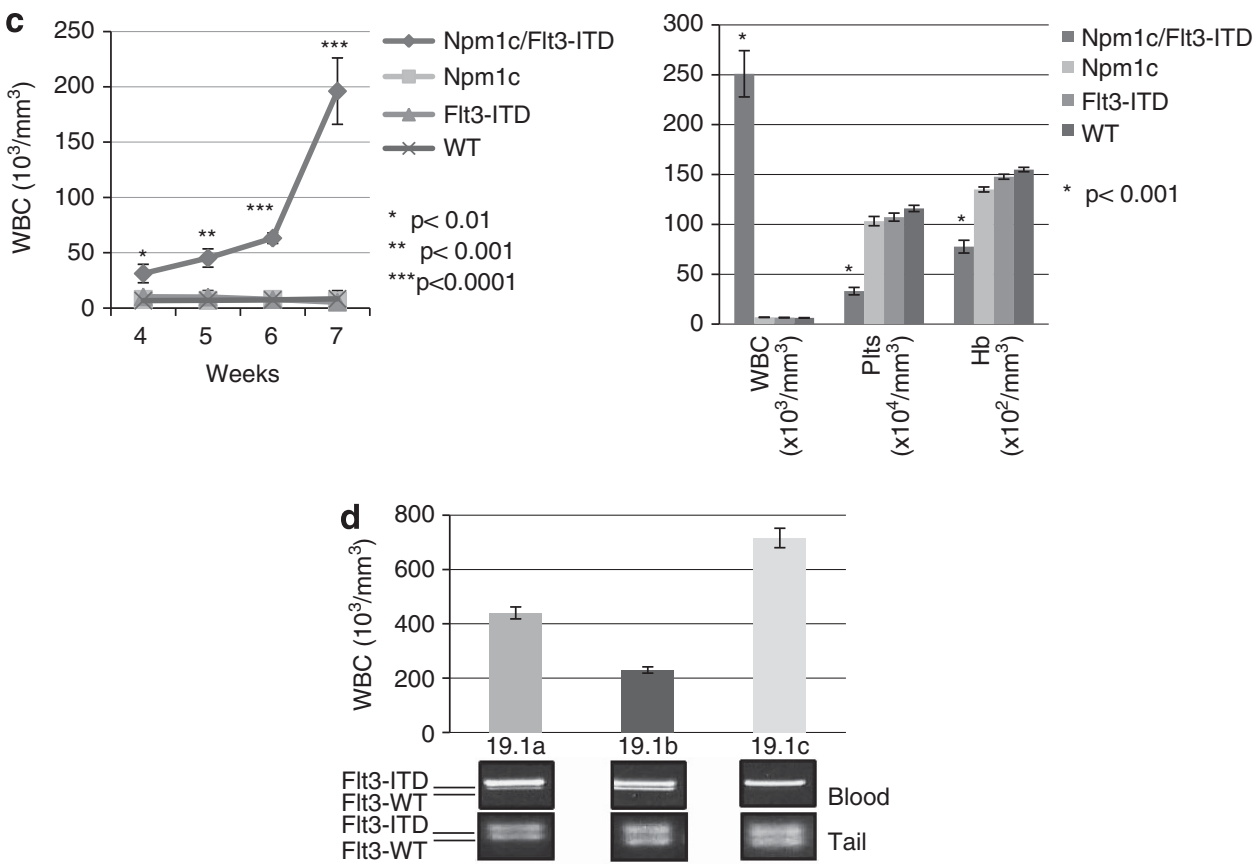

Figure 1. Npm1C and Flt3-ITD collaborate to drive rapid-onset leukemogenesis with frequent occurrence of Flt3 LOH. (a) Npm1 mutant protein (arrow) accumulates in the cytoplasm of spleen cells collected from 3-week-old Npm1c/Flt3-ITD, but not Npm1c or Flt3-ITD single-mutant mice. (b) Kaplan-Mayer survival plots showing the rapid demise of Npm1c/Flt3-ITD mice compared with all other genotypes. (c) Serial blood counts highlight a consistent explosive increase in blood leukocytes counts (WBC) between 4 and 7 weeks in Npm1c/Flt3-ITD mice (left) and the markedly abnormal WBC, platelet count (Plts) and hemoglobin concentration ( $\mathrm{Hb}$ ) of sick leukemic Npm1 /Flt3-ITD mice compared with age-matched control mice. (d) Loss of the Flt3 WT allele in blood DNA from Npm1 c/Flt3-ITD AMLs is demonstrated as loss of intensity of the Flt3-WT PCR band. By contrast, constitutional tail DNA shows no LOH. In these three littermates (19.1a-c), the extent of Flt3-LOH associates with the degree of leukocytosis ( $\mathrm{N}=$ nuclear lysate, $\mathrm{C}=$ cytoplasmic lysate, OCI-AML3 lysate as positive control, $\dagger$ nonspecific band).

age-matched control littermates, whereas the hemoglobin and platelet counts were significantly reduced (Figure 1c).

Interestingly, Npm1C/Flt3-ITD siblings/littermates often progressed to AML at different rates or developed more/less aggressive disease. To explain this observation we hypothesized that, as seen in human AML, LOH for Flt3-ITD may be responsible. We found evidence for significant spontaneous loss of the WT Flt3 allele in blood samples from Npm1c/Flt3-ITD mice and a tendency for higher blood leukocyte counts (Figure 1d) when LOH was present. $\mathrm{LOH}$ was also seen in bone marrow and spleen but not tail DNA, in keeping with somatic loss of the WT allele in leukemic cells (Figure 1d). At the time mice became sick with AML, LOH was detected in 12 of 15 spleen samples tested.

Flow cytometric analysis of blood samples demonstrated, in Npm1c/Flt3-ITD mice, a population of blasts/immature cells with low side scatter (SSC) and CD45 $5^{\mathrm{dim}}$ (Figure 2a) and a large number of single Mac1 + precursors (Figure 2b). In addition, we also observed an increased number of mature myeloid $(\mathrm{Gr} 1+$ / $\mathrm{Mac1}+$ ) cells in Npm1C/Flt3-ITD mice, indicating that any maturation block was incomplete (Figure 2b). The relative numbers of circulating $B(B 220+)$ and $T(C D 3+)$ lymphocytes were reduced (data not shown). To assay their self-renewal potential, bone marrow cells from Npm1c $(n=4)$, Flt3-ITD $(n=4)$,
WT $(n=4)$ and Npm1c/Flt3-ITD $(n=4)$ were studied in serial replating assays. Npm1c/Flt3-ITD cells gave rise to significantly more colonies at first and subsequent platings than any other genotype (Figure 2c), demonstrating a significantly increased selfrenewal potential.

Blood smears from sick mice confirmed the presence of blasts, and histological sections demonstrated widespread infiltration of solid organs by abnormal myeloid cells (Supplementary Figure S1). Cells infiltrating the bone marrow and spleen were Gr1 + /Mac1 + or $\mathrm{Gr} 1-/ \mathrm{Mac1}+$, and there were increased numbers of Mac1 + / cKit + cells compared with other genotypes (Supplementary Figure S2). Compared with single mutant and WT mice, sick Npm1c/Flt3ITD mice had marked splenomegaly $(0.95 \pm 0.27 \mathrm{~g}$ vs $0.13 \pm 0.02 \mathrm{~g}$; $P<0.0001)$ and hepatomegaly $(2.33 \pm 0.26 \mathrm{~g}$ vs $1.6 \pm 0.17 \mathrm{~g}$, $P<0.0001)$ at the time of death. Npm1c/Flt3-ITD leukemias were transplantable into both syngeneic and NOD SCID $\gamma$ mice demonstrating their true neoplastic nature (data not shown).

AML is a molecularly and clinically heterogeneous disease and recent studies have revealed that this heterogeneity is derived, to a large extent, from the specific combinations of somatic driver mutations present in individual cases. Here, we show that the combination of Npm1C and Flt3-ITD, the two most commonly co-occurring $\mathrm{AML}$ mutations, is rapidly and universally 
a

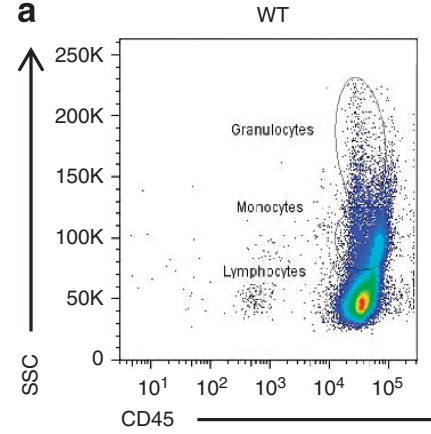

FIt3-ITD

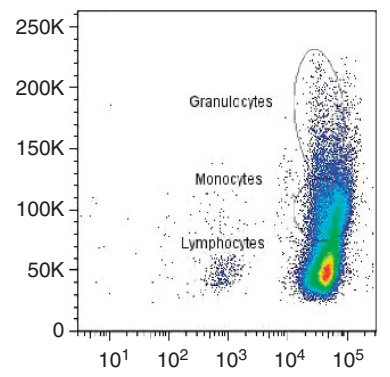

Npm1c

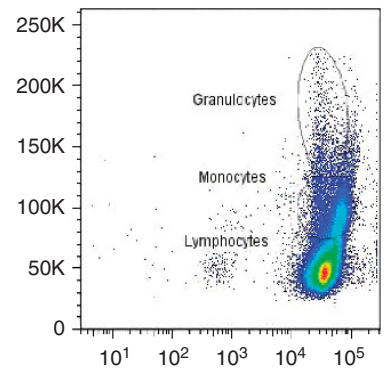

b

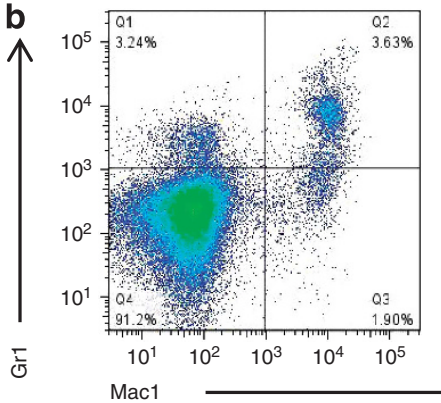

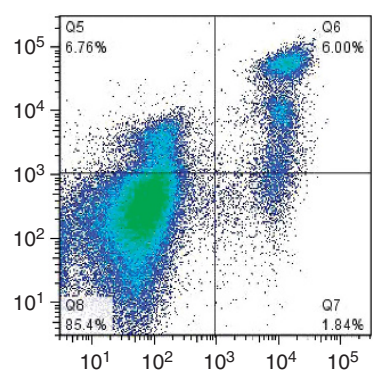
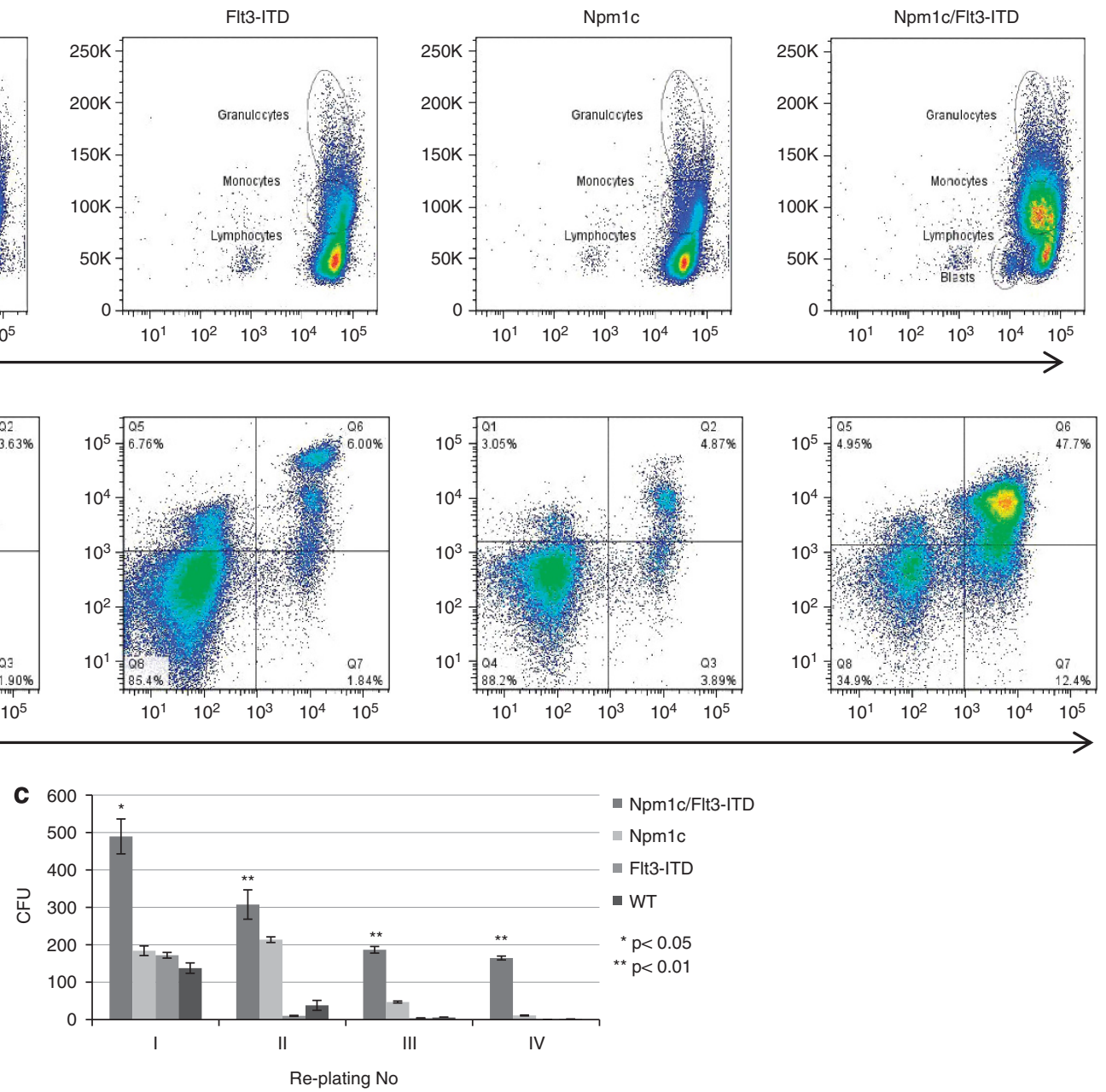

Figure 2. Expansion of circulating myeloid cells in Npm1c/Flt3-ITD mice culminating to aggressive AML. (a) Representative flow cytometric analysis of peripheral blood from 7-week-old WT, Npm1c, Flt3-ITD and Npm1c/Flt3-ITD mice shows the presence of a low SSC; CD45dim population of immature/blast cells in double mutant mice and (b) an increase in both mature granulocytic $\left(\mathrm{Gr} 1^{+} / \mathrm{Mac1} 1^{+}\right)$and monocytic $\left(\mathrm{Gr} 1^{-/ \mathrm{lo}} / \mathrm{Mac}^{+}\right)$populations. (c) Colony-forming assays of BM cells derived from WT and mutant mice showing a markedly increased replating ability of Npm1c/Flt3-ITD cells compared with other genotypes, indicative of an increased self-renewal potential. As we described before, a lesser increase in replating ability is observed with Npm1c cells.

leukemogenic in knock-in mice. These findings are particularly striking in light of the fact that, in isolation, both $\mathrm{Npm}_{1} \mathrm{c}^{4}$ and Flt3ITD ${ }^{11}$ mutations have relatively subtle effects on mouse hemopoiesis and lead to leukemia or a myeloproliferative disorder only after prolonged latencies and in a minority of mice.

What is most remarkable about our findings is the very short latency of AML in Npm1c/Flt3-ITD mice, which suggests either: (i) that the two mutations are sufficient to promote $A M L$ in this strain of mice (C57BL6/N) or (ii) that additional mutations are acquired very rapidly in the pool of cells susceptible to leukemic transformation. The later possibility is supported by the fact that at least one type of somatic mutation, namely LOH for Flt3-ITD, was frequently observed in our mouse AMLs over this short time span. We recently reported that $N p m 1 c$ can generate $A M L$ in collaboration with, amongst others, activating insertions of the GrOnc transposon in intron 9 of mouse Flt3. These insertions led to aberrant expression of a Flt3 messenger RNA predicted to code for an amino-terminal truncated version of Flt $3^{4}$ which, like Flt3-ITD, was thought to be constitutively active. Most of these murine AMLs harbored additional transposon insertions thought to be important in leukemogenesis. Thus, at this stage it appears more likely that additional mutations may be required for leukemogenesis in our Npm1c/Flt3-ITD mice, but this cannot be stated unequivocally.
In interesting contrast to our present work, a recent report demonstrated that the combination of Flt3-ITD with NUP98HOXD13 in mice led to AML after a much longer latency (median 95 days), ${ }^{14}$ despite the fact that, unlike Npm1c, NUP98-HOXD13 alone leads to a highly penetrant myelodysplastic syndrome with a high risk of leukemic transformation. This relative delay is particularly intriguing as NUP98-HOXD13 can promote leukemic transformation in association with simple overexpression of WT FLT3. ${ }^{15}$ By contrast, in two large transposon-mediated insertional mutagenesis screens, one published ${ }^{4}$ and one ongoing, we never observed transposon insertions leading to simple Flt3 overexpression amongst $>100$ mouse Npm1c + ve AMLs.

Notwithstanding the above, our observations emphasize the remarkable complementarity between Npm1c and Flt3-ITD. In the context of a stochastic model for AML pathogenesis, ${ }^{2}$ this potent molecular synergy goes some way toward explaining why NPM1C and FLT3-ITD co-occur so frequently and make the model described here a valuable tool for the study of the pathogenesis and treatment of one of the largest molecularly defined subgroups of AML.

\section{CONFLICT OF INTEREST}

The authors declare no conflict of interest. 


\section{ACKNOWLEDGEMENTS}

We are grateful to Dr Gary Gilliland for his kind donation of the Flt3-ITD mice. We thank James Bussell and the members of the Wellcome Trust Sanger Institute's Research Support Facility for help with mouse colony management, controlled procedures and daily welfare checks. We also thank the Haematooncology Diagnostic service and the Pathology Tissue Bank of Cambridge University NHS Trust for their help with sample processing for microscopy. We are grateful to the Wellcome Trust and the Kay Kendall Leukaemia Fund for funding this work and also to Leukaemia Lymphoma Research for funding related work in GV's laboratory.

All material is original research, and has neither been previously published or submitted for publication elsewhere.

\section{A Mupo ${ }^{1}$, L Celani ${ }^{1,2}$, O Dovey $^{1}$, JL Cooper ${ }^{1}$, C Grove $^{1}$, R Rad $^{1}$ P Sportoletti ${ }^{2}$, B Falini $^{2}$, A Bradley ${ }^{1}$ and GS Vassiliou ${ }^{1}$ \\ ${ }^{1}$ The Wellcome Trust Sanger Institute, Wellcome Trust Genome Campus, Hinxton, Cambridge, UK and \\ ${ }^{2}$ Hematology and Clinical Immunology Section, Department of Clinical and Experimental Medicine, University of Perugia, \\ Perugia, Italy \\ E-mail: gsv20@sanger.ac.uk}

\section{REFERENCES}

1 Grimwade D, Hills RK. Independent prognostic factors for AML outcome. Hema tology Am Soc Hematol Educ Program 2009; 1: 385-395.

2 Welch JS, Ley TJ, Link DC, Miller CA, Larson DE, Koboldt DC et al. The origin and evolution of mutations in acute myeloid leukemia. Cell 2012; 150: 264-278.

3 Patel JP, Gonen M, Figueroa ME, Fernandez H, Sun Z, Racevskis J et al. Prognostic relevance of integrated genetic profiling in acute myeloid leukemia. $N$ Engl J Med 2012; 366: 1079-1089.

4 Vassiliou GS, Cooper JL, Rad R, Li J, Rice S, Uren A et al. Mutant nucleophosmin and cooperating pathways drive leukemia initiation and progression in mice. Nat Genet 2011; 43: 470-475.

5 Sasaki M, Knobbe CB, Munger JC, Lind EF, Brenner D, Brustle A et al. IDH1(R132H) mutation increases murine haematopoietic progenitors and alters epigenetics. Nature 2012; 488: 656-659.
6 Hingorani K, Szebeni A, Olson MO. Mapping the functional domains of nucleolar protein B23. J Biol Chem 2000; 275: 24451-24457.

7 Falini B, Mecucci C, Tiacci E, Alcalay M, Rosati R, Pasqualucci L et al. Cytoplasmic nucleophosmin in acute myelogenous leukemia with a normal karyotype. $N$ Engl J Med 2005; 352: 254-266.

8 Falini B, Bolli N, Liso A, Martelli MP, Mannucci R, Pileri S et al. Altered nucleophosmin transport in acute myeloid leukaemia with mutated NPM1: molecular basis and clinical implications. Leukemia 2009; 23: 1731-1743.

9 Whitman SP, Archer KJ, Feng L, Baldus C, Becknell B, Carlson BD et al. Absence of the wild-type allele predicts poor prognosis in adult de novo acute myeloid leukemia with normal cytogenetics and the internal tandem duplication of FLT3: a cancer and leukemia group B study. Cancer Res 2001; 61: 7233-7239.

10 Kiyoi H, Towatari M, Yokota S, Hamaguchi M, Ohno R, Saito $\mathrm{H}$ et al. Internal tandem duplication of the FLT3 gene is a novel modality of elongation mutation which causes constitutive activation of the product. Leukemia 1998; 12 1333-1337.

11 Lee BH, Tothova Z, Levine RL, Anderson K, Buza-Vidas N, Cullen DE et al. FLT3 mutations confer enhanced proliferation and survival properties to multipotent progenitors in a murine model of chronic myelomonocytic leukemia. Cancer Cell 2007; 12: 367-380

12 Kemp R, Ireland H, Clayton E, Houghton C, Howard L, Winton DJ. Elimination of background recombination: somatic induction of Cre by combined transcriptional regulation and hormone binding affinity. Nucleic Acids Res 2004; 32: e92.

13 Kiyoi H, Ohno R, Ueda R, Saito H, Naoe T. Mechanism of constitutive activation of FLT3 with internal tandem duplication in the juxtamembrane domain. Oncogene 2002; 21: 2555-2563.

14 Greenblatt S, Li L, Slape C, Nguyen B, Novak R, Duffield A et al. Knock-in of a FLT3/ ITD mutation cooperates with a NUP98-HOXD13 fusion to generate acute myeloid leukemia in a mouse model. Blood 2012; 119: 2883-2894.

15 Palmqvist L, Argiropoulos B, Pineault N, Abramovich C, Sly LM, Krystal G et al. The Flt3 receptor tyrosine kinase collaborates with NUP98-HOX fusions in acute myeloid leukemia. Blood 2006; 108: 1030-1036.

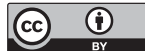

This work is licensed under a Creative Commons Attribution 3.0 Unported License. To view a copy of this license, visit http:// creativecommons.org/licenses/by/3.0/

\section{Phase I study of ON 01910.Na (Rigosertib), a multikinase PI3K inhibitor in relapsed/refractory B-cell malignancies}

\author{
Leukemia (2013) 27, 1920-1923; doi:10.1038/leu.2013.79
}

Chronic lymphocytic leukemia (CLL) and mantle cell lymphoma $(\mathrm{MCL})$ are incurable B-cell malignancies usually responsive to initial immunochemotherapy, but virtually all patients experience relapse. Salvage therapy choices for relapsed/refractory disease are often limited by resultant cytopenias and acquired drug resistance. The current priority in these B-cell malignancies, therefore, is to develop agents with novel mechanisms of action that are selective for tumor cells, overcome shared patterns of acquired drug resistance and exhibit limited toxicities.

Styrylbenzylsulfones are a new family of non-ATP-competitive anticancer agents that induce apoptosis in a variety of tumor cell lines, including those resistant to many chemotherapy agents. ${ }^{1,2}$ As a class, styrylbenzylsulfones inhibit cell cycle progression and induce mitotic arrest of tumor cells with less toxicity to normal human cells. ${ }^{3,4}$ ON 01910.Na (rigosertib) is a styryl sulfonyl compound that demonstrated inhibition of phosphatidylinositol-3-kinase (PI3K), preferentially targeting the $\mathrm{PI} 3 \mathrm{~K} \alpha$ and $\mathrm{PI} 3 \mathrm{~K} \beta$ isoforms, and triggered apoptosis via the release of cytochrome $c$ from mitochondria in MCL cell lines. ${ }^{3}$ Rigosertib's mechanism of action was initially considered to include inhibition of polo-like 1 kinase, ${ }^{4}$ but evidence for direct inhibition was not confirmed in subsequent studies ${ }^{5}$ and its antimitotic activity may rely on the phosphorylation of mitosis coordinator RanGAP1 SUMO1. ${ }^{6}$ First-in-man studies of rigosertib in solid tumors demonstrated excellent tolerability with limited hematologic toxicity. ${ }^{7}$ Rigosertib has also demonstrated preclinical and early clinical activities in myelodysplastic syndromes (MDS), ${ }^{8,9}$ and it is currently being tested in a randomized phase III trial in patients with relapsed/refractory MDS (NCT01241500).

We have previously reported that rigosertib induces rapid apoptosis in CLL cells with the relative sparing of normal B-cells and T-cells. ${ }^{10}$ We demonstrated that the in vitro activity of rigosertib involved a dual mechanism of inhibition of PI3K pathway signaling coupled with the induction of an oxidative stress response. Importantly, activity of rigosertib was equally observed against 